\title{
Efficacy comparison of multi-phase CT and hepatotropic contrast-enhanced MRI in the differential diagnosis of focal nodular hyperplasia: a prospective cohort study
}

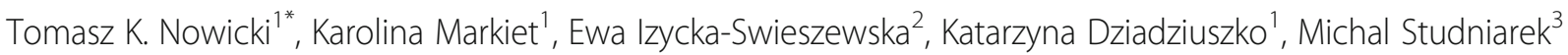
and Edyta Szurowska ${ }^{1}$

\begin{abstract}
Background: Different clinical behaviour influences the importance of differentiating focal nodular hyperplasia (FNH) from other focal liver lesions (FLLs). The aim of this study was to compare the efficacy of contrast-enhanced $\mathrm{CT}$ and $\mathrm{MRI}$ in the diagnosis of $\mathrm{FNH}$.

Methods: 157 patients with equivocal FLLs detected in ultrasonography subsequently underwent multi-phase CT and MRI with the use of hepatotropic contrast agent (Gd-BOPTA) in a 1.5 T scanner. Examinations were evaluated by three independent readers. Diagnostic efficacy of different radiological signs of FNH in both $\mathrm{CT}$ and MRI was compared and AFROC analysis was performed.
\end{abstract}

Results: 4 hepatocellular adenomas, 95 hepatocellular carcinomas, 98 hemangiomas, 138 metastases and 45 FNHs were diagnosed. In both $C T$ and MRI the radiological sign of the highest accuracy was the presence of the central scar within FNH (0.93 and 0.96 relatively). The sum of two radiological signs in MRl: homogeneous enhancement in hepatic arterial phase (HAP) and enhancing lesion in hepatobiliary phase (HBP) was characterized with high values of sensitivity (0.89), specificity (0.97), PPV (0.82), NPV (0.98) and accuracy (0.96). After inclusion of clinical data into analysis the best discriminating feature in MRI was the presence of enhancing lesion in HBP in patients without cirrhosis. In this regard, efficacy parameters increased to 1.00, 0.99, 0.94, 1.00 and 0.99 accordingly. The area under the curve in AFROC analysis of MRI performance was significantly larger than of CT ( $p=0.0145)$.

Conclusion: Gd-BOPTA-enhanced MRI is a more effective method in the differential diagnosis of FNH than multi-phase $\mathrm{CT}$.

Keywords: Focal nodular hyperplasia, Magnetic resonance imaging, Computed tomography, Differential diagnosis, Hepatobiliary phase, Gadobenate Dimeglumine, AFROC

\section{Background}

Focal nodular hyperplasia (FNH) is a benign lesion, composed of hyperplastic hepatocytes separated by fibrous septa with common central scar (see Additional file 1). FNH is most probably a reactive proliferation of hepatocytes due to preexistent vascular malformation [1].

\footnotetext{
* Correspondence: tnowicki@gumed.edu.pl

2nd Departement of Radiology, Medical University of Gdansk,

Smoluchowskiego 17, 80-214 Gdansk, Poland

Full list of author information is available at the end of the article
}

It is the next most frequent benign liver lesion following hemangioma and usually develops in unchanged liver parenchyma [2]. It is encountered in $0.3 \%-6 \%$ of the general population [3] but the incidence is increasing, partly due to progress in radiological imaging.

Different clinical behaviour and pathological features influence the importance of differentiating FNH from other hypervascular liver lesions such as hepatocellular adenoma (HCA), hepatocellular carcinoma (HCC), and hypervascular metastases as it is critical to ensure proper treatment. 
Clinical symptoms and biochemical parameters in FNH are nonspecific. Diagnosis based on ultrasonography and core needle biopsy may not be conclusive. Furthermore, correct diagnosis of FNH in computed tomography $(\mathrm{CT})$ and magnetic resonance imaging (MRI) may not be possible even in about $30 \%$ and $20 \%$ of cases respectively due to atypical radiological features $[4,5]$. However, MRI with the use of organ-specific contrast agents optimizes diagnosis [6-9]. There are two hepatotropic contrast agents available: gadoxetic acid and gadobenate dimeglumine (Gd-BOPTA). Hepatotropic contrast agents applied in MRI have a vascular-interstitial distribution during the first few minutes and are partially excreted by kidneys to urine. $3-5 \%$ of Gd-BOPTA and $50 \%$ of the gadoxetic acid is taken up by hepatocytes with normal metabolism and secreted into bile [10]. Due to contrast agent uptake by hepatic cells, it is possible to observe the enhancement of the liver parenchyma in so-called hepatobiliary phase (HBP). Enhancement persists for 1-4 h after administration of Gd-BOPTA and 20-40 min after gadoxetic acid [11]. Lesions lacking active hepatocytes are less enhancing than surrounding parenchyma or not enhancing at all. Parenchymal cells constituting FNH accumulate hepatotropic contrast agents in opposition to hepatic hemangiomas $(\mathrm{HH})$ and the majority of metastases and hepatocellular carcinomas (HCC).

To compare the efficacy of two imaging modalities (CT vs. MRI) in the assessment of multiple liver lesions in different locations a conventional receiver operating characteristic (ROC) is not sufficient. However, the alternative free-response receiver operating characteristic (AFROC) methodology incorporates lesions location and confidence level of a reader [12]. AFROC curve is a plot of the fraction of correctly diagnosed lesions in their true location (lesion location fraction, LLF) and false positive fraction (FPF) [12].

The aim of this study is to compare the efficacy of multi-phase multi-detector CT and MRI with the use of hepatotropic contrast agent Gd-BOPTA in the differentiation of FNH from other focal liver lesions (FLLs) in patients with equivocal foci detected in ultrasonography.

\section{Methods}

In this prospective study, we included 207 patients with equivocal FLLs detected in ultrasonography and no contraindications to CT and MRI including administration of iodine contrast agent and Gd-BOPTA respectively. Multi-phase multi-detector CT and dynamic contrastenhanced liver MRI with the use of hepatotropic contrast agent Gd-BOPTA were performed within 4 weeks. 43 patients did not show for either initial MRI or follow-up. Seven patients were excluded due to artifacts in MRI preventing further evaluation of lesions (Fig. 1).

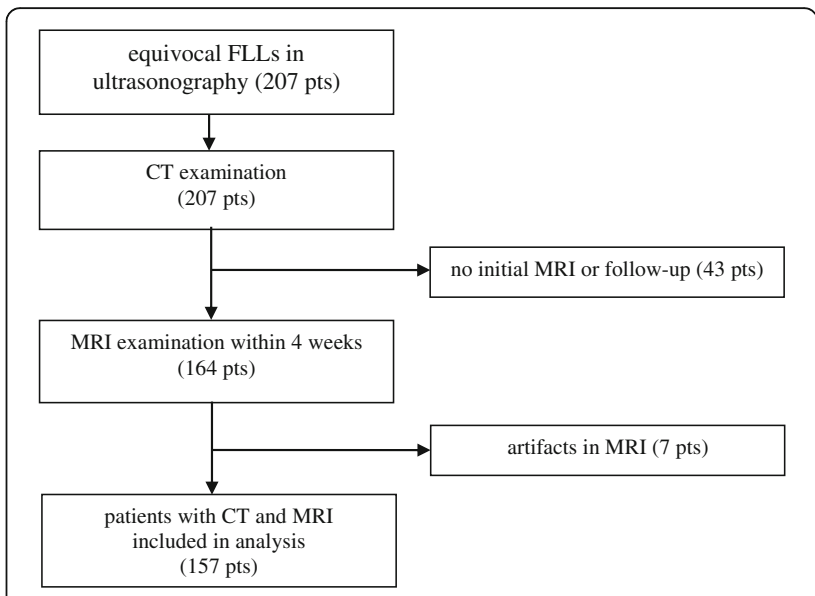

Fig. 1 Flowchart of patients' inclusion in the analysis. FLLs - focal liver lesions, pts - patients

\section{CT \& MRI protocol}

CT examination consisted of non-enhanced and contrastenhanced phases. Iodine contrast agent was administered by power injector at the flow rate of $4 \mathrm{~mL} / \mathrm{s}$ and hepatic arterial phase (HAP), portal venous phase (PVP) and equilibrium phase (EP) were acquired. In order to optimize the parameters of multiphase examination, bolus tracking technique was applied. CT section thickness was 2-2.5 mm, pitch - 1.5 .

Liver MRI examination was performed with the use of a $1.5 \mathrm{~T}$ scanner and a phase array coil or body coil. Non-contrast examination included spin echo sequence (TR/TE - 303/12 ms, scan time - $17 \mathrm{~s}$ ) and single shot fast spin echo sequences $(18,000 / 80 \mathrm{~ms}, 17 \mathrm{~s})$ in $\mathrm{T} 1$ and T2-weighted images in three perpendicular planes as well as sequences with fat saturation - fast spin echo sequences $(6500 / 116.8 \mathrm{~ms}, 22 \mathrm{~s})$ and spoiled gradient echo sequence $\left(150 / 2.24 \mathrm{~ms}, 12 \mathrm{~s}\right.$, flip angle of $\left.90^{\circ}\right)$. Section thickness was $5 \mathrm{~mm}$, intersection gap $-1 \mathrm{~mm}$, FOV $35 \times 32 \mathrm{~cm}$, matrix $-256 \times 256$. Gd-BOPTA was administered in manual bolus through a venous catheter in a dose of $0.1 \mathrm{mmol} / \mathrm{kg}$ per body weight. HAP, PVP and EP were obtained after 25,60 and 180 s post-contrast agent administration respectively. HBP in spoiled gradient echo and spin echo sequences was obtained $60 \mathrm{~min}$ after contrast agent administration. Phase-encoding direction was anterior-posterior for all sequences. All images were acquired with breath-hold technique.

\section{Image analysis}

CT and MRI were evaluated by three independent readers with at least 5 years of experience in abdominal imaging. Readers, who were blind to patients' clinical data and ultrasonography reports analyzed CT examination in the first session and MRI in the second session. 
Number, longest diameter in axial plane and localization of lesions within liver segments, their density in $\mathrm{CT}$ and signal intensity on T1- and T2-weighted images were evaluated. Signal intensity was assessed in comparison to the adjacent liver parenchyma in $\mathrm{T} 1$ - and T2weighted images. The pattern of contrast enhancement in consecutive phases and the presence of central scar were further assessed. In HAP peripheral (ring-like or peripheral nodular enhancement) or central (homogeneous or heterogeneous) enhancement patterns were registered. In PVP enhancement pattern was divided into following subtypes: equal to the liver, ring enhancement, centripetal enhancement, homogeneous enhancement, heterogeneous enhancement or absence of enhancement (wash-out). In EP and HBP density and/or signal intensity of the lesions in comparison with the adjacent liver parenchyma was assessed.

Lastly, readers chose the most likely diagnosis and provided confidence level in both CT and MRI. The following diagnoses were proposed: $\mathrm{HH}, \mathrm{HCA}, \mathrm{FNH}$, $\mathrm{HCC}$ and metastasis. The confidence level was given according to a five-point scale: 5 - very likely, 4 - likely, 3 - equivocal, 2 - unlikely, 1 - very unlikely.

\section{Statistical analysis}

For the purpose of analysis, patients were divided into two groups according to the final diagnosis: one group consisted of patients with FNH (FNH group) and the other of patients with remaining FLLs (non-FNH group). The mean of patients age and lesion size was calculated in both groups. The $X^{2}$ and Mann-Whitney $U$ tests were used for population and lesion size analysis.

To establish diagnostic criteria enabling recognition of FNH on the basis of CT and MRI, statistically more frequent radiological signs were selected in the FNH group. Additional clinical data such as lack of liver cirrhosis and neoplastic disease in anamnesis were evaluated in accordance with the definition of FNH [13]. Sensitivity, specificity, PPV, NPV and accuracy for given radiological signs and clinical features differentiating $\mathrm{FNH}$ from other FLLs were calculated.

Subsequently, the performance of different features was assessed by means of Cochran's Q test and post hoc analysis with McNemar's test and Bonferroni-Hochberg's $p$-value correction for multiple comparisons.

In the end, the efficacy of characterization of FNH in multi-phase CT and Gd-BOPTA-enhanced MRI was compared with the implementation of lesions' localization and readers' confidence level by means of AFROC analysis. The area under the curve (AUC) for CT and MRI was compared with F-statistics. Statistical analyses were computed with Statistica 12 software (StatSoft Inc., Tulsa, OK, USA) and JAFROC 4.2.1 [12, 14]. A $p$ value less than 0.05 was considered significant.
The research was approved by The Independent Bioethics Committee for Scientific Research. All patients gave their informed written consent to participate.

\section{Results}

157 patients who underwent both CT and MRI were included in the analysis.

In 89 patients final diagnosis was based on the histopathological examination: 21 patients were diagnosed with FNH, $35-$ HCC, $4-$ HCA and 22 with metastases. In the remaining 68 patients, the final diagnosis was based upon clinical and imaging follow-up revealing $\mathrm{HH}$ in 36 patients, $\mathrm{FNH}$ in 18 patients and liver metastases in 21 patients. In 15 patients diagnosed with $\mathrm{FNH}$, the central scar was seen in histopathological examination. In the rest of FNH specimens areas of congestion were observed.

Table 1 lists all clinical symptoms for non-FNH and $\mathrm{FNH}$ group that lead to initial ultrasound examination and subsequently examined in CT and MRI.

In 38 patients liver cirrhosis was recognized on the basis of core needle biopsy, 35 of those patients were diagnosed with $\mathrm{HCC}$, one with renal cancer metastasis and two with colorectal cancer metastases.

In FNH-group, slight elevation of serum GGTP level was seen in 10 patients, in all remaining cases biochemical examinations showed no significant changes, none of the patients was diagnosed with cirrhosis. In 6 patients with oncologic history, FLLs were detected at the followup abdominal ultrasonography. In 21 cases FNHs were detected incidentally (Table 1).

Patients characteristics in FNH group and non-FNH group are listed in Table 2. The first group included 118 patients with 335 non-FNH foci (Table 3). The second group included 39 subjects with $45 \mathrm{FNH}$ foci.

Women were significantly more frequent in $\mathrm{FNH}$ - group $(31 / 39)$ than in non-FNH group (64/118), $x^{2}=7.82$, $p=0.0052$. Statistically significant difference was also seen in patients' age and lesion diameter: in FNH-group the mean age was 36 years $(18 \div 56)$ vs 56 years $(21 \div 79)$ in

Table 1 The indication for performing ultrasonography in patients examined by CT and MRI $(n=157)$

\begin{tabular}{lll}
\hline & non-FNH & FNH \\
\hline $\begin{array}{lll}\text { digestive tract carcinoma } \\
\text { colorectal/pancreatic/gastric carcinoma) }\end{array}$ & $30 / 7 / 1$ & $0 / 2 / 0$ \\
renal carcinoma & 6 & 1 \\
melanoma malignum & 1 & 0 \\
other neoplasm & 6 & $1^{*}$ \\
hepatic cirrhosis & 38 & 0 \\
abdominal pain & 13 & 14 \\
no symptoms & 16 & 21 \\
\hline
\end{tabular}

*breast carcinoma 
Table $\mathbf{2}$ Characteristics of the non-FNH and FNH group

\begin{tabular}{lll}
\hline & non-FNH & FNH \\
\hline number of patients & 118 & 39 \\
number of foci & 335 & 45 \\
average age & 57 & 36 \\
male/female ratio & $54 / 64$ & $8 / 31$ \\
\hline
\end{tabular}

non-FNH group $(\mathrm{Z}=6.97, p<0.0001)$, in FNH-group the mean diameter of a focus was $37 \mathrm{~mm}(10 \div 85 \mathrm{~mm})$ vs $29 \mathrm{~mm}(5 \div 80 \mathrm{~mm})$ in non-FNH group $(\mathrm{Z}=-4.03, p<$ 0.0001).

Interobserver reproducibility and agreement for all parameters were high with Kappa values of $0.85 \div 1.0$.

In analyzed patients, CT revealed 335 lesions and MRI - 380. MRI discovered 21 more HH, 14 more HCC and 10 more metastases. Both in CT and MRI the number of diagnosed FNH foci was equal. 6 patients had bifocal FNH.

Radiological signs (Fig. 2 and Additional file 2, Additional file 3 and Additional file 4) appearing statistically more often in FNH than in other FLLs in $\mathrm{CT}$ and/or MRI included:

1. homogeneous enhancement in HAP in both CT and MRI (38/40 FNH foci and 67/84 other lesions in CT and MRI relatively),

2. presence of central scar (24/33 FNH foci and 3/5 $\mathrm{HH}$ in $\mathrm{CT}$ and MRI accordingly),

3. enhancement similar to that of the liver in PVP $(36 / 37$ FNH foci and 59/72 other lesions in CT and MRI relatively),

4. enhancement similar to that of the liver in EP (45/45 FNH foci and 164/204 other lesions in CT and MRI relatively),

5. density similar to that of the liver in non-enhanced CT (40 FNH foci and 111 other lesions),

6. isointense or hyperintense signal of the focus in comparison to the adjacent liver parenchyma in HBP (all FNH foci and 21 other lesions),

7. intensity similar to that of the liver on $\mathrm{T} 1$ - and T2-weighted images in MRI (20 FNH foci and 53 other lesions on T1-weighted images and $38 \mathrm{FNH}$ foci and 49 other lesions on T2-weighted images).

Table 3 Final diagnosis in the non-FNH group

\begin{tabular}{lll}
\hline histopathological diagnosis & number of patients & number of foci \\
\hline HCC & 35 & 95 \\
HH & 36 & 98 \\
metastases & 43 & 138 \\
HCA & 4 & 4
\end{tabular}

HCA Hepatocellular adenoma, $H C C$ Hepatocellular carcinoma, $H H$ Hepatic hemangioma
Diagnostic efficacy of above mentioned radiological signs and other analyzed features is presented in Table 4.

Presence of central scar allows differentiating $\mathrm{FNH}$ from other FLLs only in 53\% of cases in CT and 73\% in MRI (accuracy of 93\% and 96\% relatively). In both CT and MRI foci with visible scar were significantly larger (mean of $27 \mathrm{~mm}, \mathrm{SD}=13 \mathrm{~mm}$ vs. $45 \mathrm{~mm}, \mathrm{SD}=12 \mathrm{~mm}$ in CT and $20 \mathrm{~mm}, \mathrm{SD}=9 \mathrm{~mm}$ vs. $43 \mathrm{~mm}, \mathrm{SD}=13 \mathrm{~mm}$ in MRI, $p<0.001$ ).

Homogeneous enhancement in HAP in both CT and MRI is a feature of a good diagnostic efficacy (78\% and $77 \%$ accordingly). However, low positive predictive value (PPV) (36\% and 32\% in CT and MRI accordingly) strongly decreases its clinical usefulness.

Feature characterized by the highest values of sensitivity (100\%) and specificity (94\%) in the diagnosis of FNH in MRI was the enhancement of the lesion in HBP. In all cases of $\mathrm{FNH}$, the focus was isointense ( 42 foci) or hyperintense (3 foci) in comparison to the adjacent liver parenchyma in HBP. The same enhancement pattern in HBP as in $\mathrm{FNH}$ observed in case of $21 \mathrm{HCC}$ foci (isointense foci) resulted in PPV of $68 \%$ for this feature. Moreover, 4 of the enhancing in HBP HCC foci presented with homogeneous enhancement in HAP and were isointense in PVP and EP. However, in all those cases patients suffered from cirrhosis.

To increase the efficacy parameter further analysis was conducted on logic sums of different radiological signs and clinical data. This analysis was performed separately for CT and MRI (Table 4).

Based on MRI study we stated that simultaneous occurrence of two radiological signs (non-hypointense enhancement in HBP and homogeneous enhancement in HAP) was a criterion enabling the diagnosis of FNH with PPV of $82 \%$. Furthermore, after exclusion of cirrhotic patients, the efficacy of HBP reached very high values: sensitivity of $100 \%$, specificity of $99 \%$, PPV of $94 \%$, the negative prognostic value of $100 \%$ and accuracy of $100 \%$ (Table 4).

By means of Cochran's Q test, the most efficient radiological signs were compared and significant differences were stated both in CT and in MRI $(p<0.001)$.

In post hoc analysis the McNemar's test with Bonferroni-Hochberg's correction was performed to compare individual radiological signs and logic sums. Presence of central scar and the logic sum of homogeneous enhancement in HAP and presence of central scar proved to diagnose FNH better than the logic sum of enhancement pattern in HAP and PVP $(p=0.0072$ and $p=0.0006$ accordingly). Logic sum of enhancement pattern of HAP and PVP and exclusion of cirrhosis recognized FNH better than the logic sum of homogeneous enhancement in HAP and presence of central scar $(p=0.006)$. However, the logic sum of enhancement pattern of HAP and PVP and exclusion of cirrhotic patients did not perform 


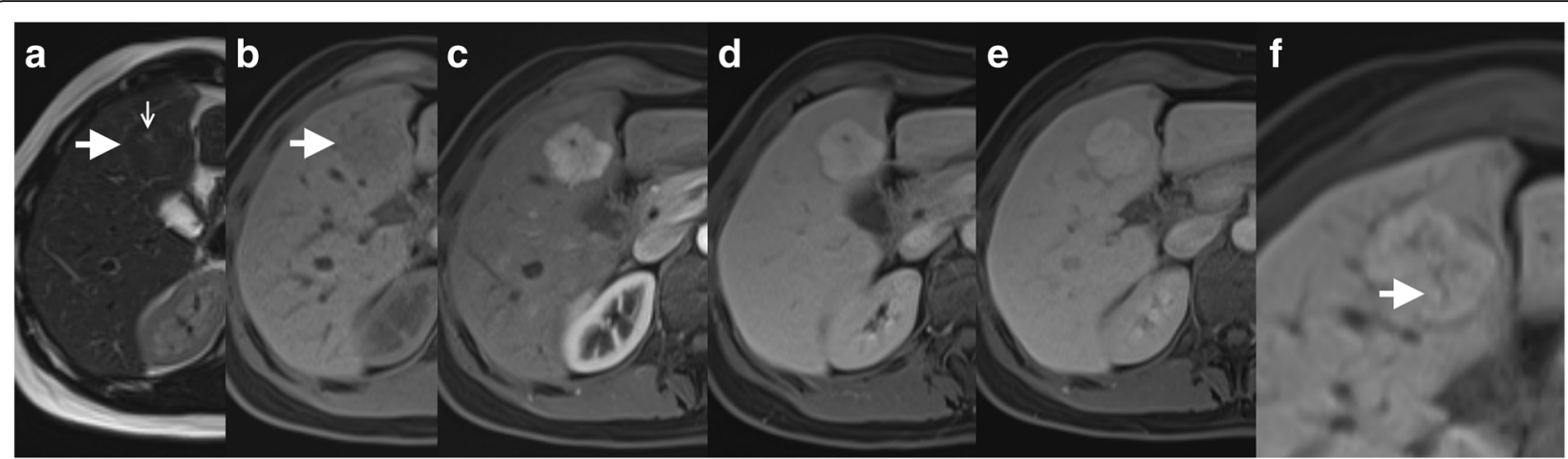

Fig. 2 MRI examination of a 33-year-old female; all the images are in the axial plane. Fig. a and b present slightly hypointense FNH in segment IV of the liver (arrow) on T2- and T1-weighted images. Central scar (small arrow) is hyperintense on T2-weighted images (Fig a). In hepatic arterial phase, the lobulated lesion presents strong enhancement (Fig. C). In portal venous phase (Fig. d) and equilibrium phase (Fig. e) the lesion stays hyperintense, however, the enhancement is weaker than in hepatic arterial phase. In hepatobiliary phase (Fig. f) the lesion is hyperintense and enhancement of the scar is visible (arrow)

significantly better than the presence of central scar alone $(p=0.054)$ or the logic sum of enhancement pattern in HAP and PVP $(p=0.0824)$. All the results are presented in Additional file 5. In MRI the logic sum of enhancement pattern in HAP and HBP after exclusion of cirrhotic patients determined FNH better than enhancement pattern in HBP alone $(p<0.0001)$, the logic sum of enhancement pattern in HAP and HBP $(p<0.0001)$ and the logic sum of enhancement pattern in HAP and HBP and presence of central scar

Table 4 Diagnostic efficiency of radiological findings in CT and MRI and their logical sum

\begin{tabular}{|c|c|c|c|c|c|c|}
\hline & & sensitivity & specificity & PPV & NPV & accuracy \\
\hline \multirow[t]{9}{*}{$\mathrm{CT}$} & isodense focus in NECT & 0.93 & 0.74 & 0.36 & 0.99 & 0.76 \\
\hline & homogeneous enhancement in HAP & 0.84 & 0.77 & 0.36 & 0.97 & 0.78 \\
\hline & isodense to liver enhancement in PVP & 0.80 & 0.80 & 0.38 & 0.96 & 0.80 \\
\hline & isodense to liver enhancement in EP & 1.00 & 0.43 & 0.22 & 1.00 & 0.51 \\
\hline & central scar & 0.53 & 0.99 & 0.89 & 0.93 & 0.93 \\
\hline & homogeneous enhancement in HAP and central scar & 0.47 & 0.99 & 0.88 & 0.93 & 0.93 \\
\hline & homogeneous enhancement in HAP and isodense to liver enhancement in PVP & 0.71 & 0.95 & 0.67 & 0.96 & 0.92 \\
\hline & homogeneous enhancement in HAP and isodense to liver enhancement in PVP no cirrhosis & 0.71 & 0.97 & 0.76 & 0.96 & 0.94 \\
\hline & $\begin{array}{l}\text { homogeneous enhancement in HAP and isodense to liver enhancement in PVP no neoplastic } \\
\text { disease in anamnesis }\end{array}$ & 0.55 & 0.83 & 0.29 & 0.94 & 0.80 \\
\hline \multirow[t]{12}{*}{ MRI } & isointense focus in T1 W & 0.44 & 0.84 & 0.27 & 0.92 & 0.79 \\
\hline & isointense focus in $\mathrm{T} 2 \mathrm{~W}$ & 0.84 & 0.85 & 0.44 & 0.98 & 0.85 \\
\hline & central scar & 0.73 & 0.99 & 0.87 & 0.96 & 0.96 \\
\hline & homogeneous enhancement in HAP & 0.89 & 0.75 & 0.32 & 0.98 & 0.77 \\
\hline & isointense to liver enhancement in PVP & 0.82 & 0.79 & 0.34 & 0.97 & 0.79 \\
\hline & non-hypointense focus in HBP & 1.00 & 0.94 & 0.68 & 1.00 & 0.94 \\
\hline & homogeneous enhancement in HAP non-hypointense focus in HBP & 0.89 & 0.97 & 0.82 & 0.98 & 0.96 \\
\hline & homogeneous enhancement in HAP non-hypointense focus in HBP central scar & 0.64 & 1.00 & 1.00 & 0.95 & 0.96 \\
\hline & non-hypointense focus in HBP no neoplastic disease in anamnesis & 0.55 & 0.93 & 0.56 & 0.92 & 0.87 \\
\hline & non-hypointense focus in HBP no cirrhosis & 1.00 & 0.99 & 0.94 & 1.00 & 0.99 \\
\hline & homogeneous enhancement in HAP no cirrhosis & 0.89 & 0.83 & 0.39 & 0.99 & 0.84 \\
\hline & homogeneous enhancement in HAP non-hypointense focus in HBP no cirrhosis & 0.89 & 1.00 & 1.00 & 0.99 & 0.99 \\
\hline
\end{tabular}

The logic sum of radiological findings with the highest diagnostic efficiency in italic. HAP Hepatic arterial phase, HBP Hepatobiliary phase, NPV Negative predictive value, NECT Non-enhanced computed tomography, PVP Portal venous phase, PPV Positive predictive value, $T 1 W$ T1-weighted images, $T 2$ W T2-weighted images 
$(p=0.0037)$. Though, the logic sum of enhancement pattern in HAP and HBP after exclusion of cirrhotic patients qualified $\mathrm{FNH}$ worse than enhancement pattern in HBP after exclusion of cirrhotic patients $(p=0.0148)$. All the results are presented in Additional file 6.

In AFROC analysis AUC for CT was significantly smaller than for MRI for each reader (Table 5). The mean AUC for CT examination was 0.934 (standard error 0.009, 95\% confidence interval $0.9164 \div 0.951$ ) and for MRI examination 0.941 (SE 0.008, 95\% CI 0.924 $\div 0.957$ ), (Fig. 3). The difference between AUCs for CT and MRI reached -0.007 (SE $0.003,95 \%$ CI $-0.012 \div-0.001$ ), was statistically significant $(p=0.0145)$ and in favour of MRI examination.

The confidence level of 5 and 4 (very likely and likely) was considered as a positive diagnosis of FNH. In CT the 1st reader rated 33 lesions as FNH (73\%, 32 lesions ranged as very likely and single lesion as likely to be FNH), the 2nd reader - 32 lesions (71\%, 30 lesions - very likely and 2 lesions - likely) and the 3rd reader - 32 lesions (71\%, 31 lesions - very likely and single lesion - likely). In MRI the 1st reader rated 44 lesions as FNH $(98 \%, 37$ lesions - very likely and 7 lesions - likely), the 2nd reader - 44 lesions (98\%, 32 lesions - very likely and 12 lesions - likely) and the 3rd reader - 41 lesions (91\%, 34 lesions - very likely and 7 lesions - likely).

In CT there were six false-negative lesions for the 1st reader (13\%), one for the 2 nd reader $(2 \%)$ and two for the 3 rd reader (4\%, mean of $6.7 \%)$. In MRI there were only two false-negative lesions for the 1st reader (4\%). The 2nd and 3rd reader diagnosed all FNH lesions correctly (mean $1.5 \%$ ). All the false-negative lesions were misdiagnosed as HCC.In CT there were four HCC lesions and one metastasis misinterpreted as FNH by the 2nd reader. The 3rd reader also misinterpreted four HCC lesions as $\mathrm{FNH}$. There were no false-positive lesions for the 1st reader. However, only the 1st reader misdiagnosed two HCC lesions and one HCA lesion as FNH in MRI. The misdiagnosed HCA had lobulated shape and showed enhancement in HAP and HBP, therefore mimicking FNH.

In the retrospective analysis, the readers discovered 31 of 41 missed lesions (69\%) in CT examinations. The missed lesions were HH (12 lesions), HCC (11) and metastases (8). The decision was based on a consensus of the three readers.

Table $\mathbf{5}$ The area under the curve in AFROC analysis for each reader in $C T$ and $M R I$

\begin{tabular}{lll}
\hline & CT & MRI \\
\hline 1st reader & 0.936 & 0.942 \\
2nd reader & 0.933 & 0.941 \\
3rd reader & 0.933 & 0.939 \\
mean AUC & 0.934 & 0.941 \\
\hline AUC Area under the curve & &
\end{tabular}

AUC Area under the curve

\section{Discussion}

In our study, as well as in available literature, FNH occurs most often in women in the 3rd-5th decades of life $[1,3]$. The majority of our cases $(70 \%)$ were females and mean age was 36 years. In 13\% of the patients, FNH was bifocal, and this frequency is similar to the other studies where multi-focal lesions were reported in up to $15 \%$ to $20 \%[3,15,16]$. FNH is also 10 times more common than HCA [17] and in our study, we diagnosed 4 HCA and $45 \mathrm{FNH}$. In the group of patients with $\mathrm{FNH}$, over half of the patients were asymptomatic, one-third of the patients complained of abdominal pain, $10 \%$ had a neoplastic disease with a primary focus localized outside the liver, none of the patients suffered from liver cirrhosis (Table 1). Biochemical parameters, except for a slight elevation of GGTP activity in 10 patients, did not show any changes. Similar serum GGTP level elevation was also observed by Cherqui et al. [4]. To summarize, the clinical picture of our study group did not differ from the literature data.

In our material, only $53 \%$ of lesions showed a central scar in CT. Some authors emphasize that presence of a central scar is typical for FNH foci larger than $3 \mathrm{~cm}[16,18]$. The relation between the diameter of the lesion and presence of a central scar is confirmed by correlating radiological and microscopic images $[19,20]$. Comparable to our study, Brancatelli et al. [16] observed a central scar in CT in half of the subjects in a group of 124 FNH (mean diameter of $41 \mathrm{~mm}$ ), less often in lesions under $40 \mathrm{~mm}$. In our study mean diameter of FNH with the scar was significantly larger than of foci without visible scar both in CT and MRI. Other authors reported the presence of a central scar in $75 \%$ of the cases in HAP in MRI, similar to our study (73\%). On the other hand, lack of central scar is an atypical radiological manifestation of $\mathrm{FNH}$, complicating the diagnosis $[19,20]$. Our results show that presence of a central scar has high diagnostic specificity, however, the low sensitivity limits its usefulness considerably.

The use of contrast agents allows showing enhancement differences between focal lesions and normal liver parenchyma $[5,6,8,9]$. Focal lesions such as FNH, hepatocellular carcinoma (HCC) and metastases from kidney or endocrine tumours present abundant vascularization. Most metastases, mainly from the gastrointestinal tract, are typically hypovascular. Generally, evaluation of vascularization of lesions in contrast-enhanced CT and MRI is comparable. In our material, agreement rate of enhancement pattern assessment of particular foci in CT and MRI was $94 \%$. Homogeneous enhancement in HAP was visible in $84 \%$ of FHN lesions in CT and $89 \%$ in MRI. Those findings concord with results of other authors [21, 22]. Comparable percent of FNH lesions showed enhancement similar to the adjacent liver parenchyma in PVP. However, enhancement pattern in HAP and PVP have very low 

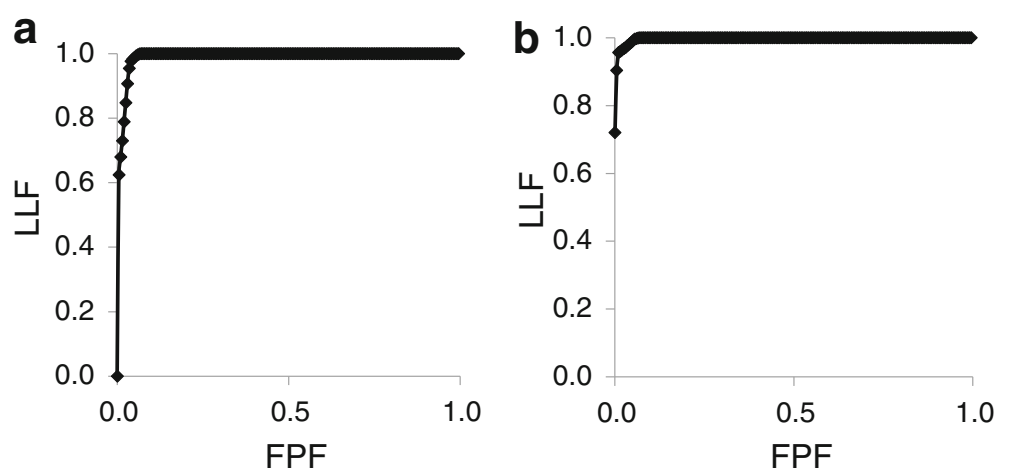

Fig. 3 Mean AFROC plot for multi-phase multi-detector CT (a) and Gd-BOPTA-enhanced MRI (b) in the diagnosis of FNH. LLF - lesion location fraction, FPF - false positive fraction

PPV (32-38\%) and the logic sum of the enhancement pattern in HAP and PVP does not improve PPV sufficiently (67\%). Moreover, the presence of central scar proved to be a better diagnostic feature than the enhancement pattern in both HAP and PVP. Only after exclusion of cirrhotic patients, diagnostic efficacy of the enhancement pattern in HAP and PVP proved to be comparable with that of a presence of central scar. This data demonstrate a need for more effective diagnostic methods in the differentiation of $\mathrm{FNH}$.

Gd-BOPTA enables detection of lesions containing active hepatocytes. Usefulness of hepato-specific contrastenhanced MRI in recognition of liver tumours has been well described [6-8].

According to Kim et al., FNH is typically a focus presenting slight enhancement in HBP with a hypointense central scar [21]. In our material, the majority of FNH foci (93\%) was isointense with the surrounding liver parenchyma in HBP and only 3 large lesions $(\geq 35 \mathrm{~mm}$ ) showed a discrete increase of the signal. All FNH foci presented with the enhancement pattern typical for the unchanged hepatocyte and were never hypointense.

In comparison, 4 adenomas, often requiring differentiation with $\mathrm{FNH}$, showed weaker enhancement than the liver parenchyma in HBP what made them unequivocally excluded from further differential diagnosis. A similar observation was made by Grazioli et al. [8] and this is most probably due to the presence of regressive changes in adenomas and lack of bile ducts. However, Roux et al. in the analysis of 27 HCA lesions found $44 \%$ of them enhancing in HBP [23]. Our material is too small to compare with that collected by Roux et al.

On the other hand, both in the study of Kim et al. [24] and in ours, malignant lesions (HCC) were found among isointense foci in HBP and constituted $22 \%$ of all HCC lesions. Huppertz et al. reported that some highly differentiated HCC can enhance similarly to normal hepatocytes in HBP [25].
The liver-specific phase of MRI in the diagnosis of FNH had satisfactory sensitivity and specificity, only PPV parameter is of $68 \%$ (Table 4). However, the sum of two radiological signs: non-hypointense lesion in HBP and homogeneous enhancement in HAP, established as criterion enabling the diagnosis of $\mathrm{FNH}$, was characterized by a higher value of PPV (82\%). HCC foci, that show isointensity in HBP and strongly enhance in HAP, are a limitation of Gd-BOPTA-enhanced MRI.

In case of good clinical and radiological cooperation in interdisciplinary teams, improved diagnostics of FLLs is possible with evaluation of additional clinical data (Table 4). Although neoplastic disease in anamnesis is twice more frequent in patients in the non-FNH group, this does not influence differential diagnostics of FNH. However, patients with liver cirrhosis were not suspected of FNH [13]. Finally, after exclusion of cirrhotic patients, presence of non-hypointense (iso- or hyperintense) foci in HBP proved to be the most accurate feature in FNH diagnosis. Parameters of diagnostic efficacy, including PPV, range from $94 \%$ to $100 \%$ (Table 4). Introduction of these diagnostic parameters gave only three false positive results (non-cirrhotic patient with a small isointense focus in HBP and final diagnosis of HCC). A non-hypointense lesion in HBP after exclusion of cirrhotic patients turned out to diagnose statistically better than other features or their logic sums beside the logic sum of homogeneous enhancement in HAP and a non-hypointense lesion in HBP. According to McNemar's test, both features diagnosed FNH similarly. However, enhancement pattern in HBP after exclusion of cirrhotic patients showed higher efficacy parameters (Table 4).

In AFROC analysis AUC for MRI was significantly larger than for CT. The authors did not find any other study applying AFROC analysis to compare the efficacy of multi-phase CT and hepatotropic contrast-enhanced MRI in the diagnosis of FNH. Chung et al. compared the efficacy of multi-phase $\mathrm{CT}$ and hepatotropic 
contrast-enhanced MRI in the diagnosis of FFL but assessed only efficacy parameters [26]. Chung et al. concluded that MRI may provide more certain diagnosis, especially in case of FNH. Soussan et al. collected data concerning confidence level of contrast-enhanced ultrasonography in comparison to MRI in the diagnosis of FFLs but did not perform FROC or AFROC analysis [27]. Sofue et al. compared the diagnostic performance of contrast-enhanced CT and a combination of contrastenhanced CT and hepatotropic contrasted-enhanced MRI with use of AFROC analysis [28] with the conclusion that combination of CT and MRI was more effective than CT alone.

This study has few limitations. Firstly, diffusion-weighted imaging in MRI was not included in the analysis. However, the study was designed to assess the efficacy of morphological and contrast-enhanced examinations only. Secondly, not all lesions were histologically proven. In $43 \%$ of patients, the final diagnosis was based upon clinical and radiological follow-up. This group contained all $\mathrm{HH}$ cases and almost half of FNH cases, where the biopsy was not possible or necessary. Thirdly, besides HCA the studied group does not include rare entities like fibrolamellar carcinoma, nodular regenerative hyperplasia or cholangiocarcinoma. Although we included incidental findings and the examined group is relatively large, we did not encounter those rare neoplasms. Finally, although we have shown that MRI has higher efficacy in the diagnosis of FNH than CT, one has to take into consideration the cost efficiency of those examinations. This issue goes beyond the scope of this paper.

In the end, it has to be emphasized, that in course of cirrhosis if a new FLL is found, it is unlikely to be FNH. In such a case, even if the lesion presents radiological features of FNH, a liver biopsy should be performed.

\section{Conclusion}

Assessment of dynamic contrast-enhanced MRI with hepatobiliary phase after Gd-BOPTA administration is a useful diagnostic tool. The only limitation of this method is small and/or highly differentiated foci of HCC, strongly enhancing after contrast agent administration in HAP and isointense in HBP. It can be avoided thanks to the cooperation of referring physician and radiologist and excluding patients with known liver cirrhosis. MRI examination with the administration of hepatotropic contrast agent is a more effective method in the differential diagnosis of FNH than multi-phase multi-detector CT. Hepatotropic compounds enable assessment of both vascularization of focal changes and hepatocyte function in the course of one examination (extracellular phase and liver-specific study in the one-stop-shop examination).

\section{Additional files}

Additional file 1: The microscopic image of $\mathrm{FNH}$ with characteristic central scar $(\times 20)$. (TIFF $5111 \mathrm{~kb})$

Additional file 2: $C T$ and MRI images of FNH in segment IVB of the liver. Fig. a. Axial $\subset$ image in hepatic arterial phase shows typical intensive homogeneous enhancement of the lesion with characteristic hypodense central scar (arrow). The lesion is isodense to normal liver parenchyma in the non-contrast examination (Fig. b) and in equilibrium phase (fig. c), but slightly hyperdense in portal venous phase (Fig. d). Fig. e. T1-weighted contrastedenhanced MRI in hepatic arterial phase shows typical enhancement pattern of FNH - intensive homogeneous enhancement and hypointensive central scar (arrow). Fig. f. Hepatobiliary phase confirms the diagnosis of $\mathrm{FNH}$ presenting stronger enhancement of FNH than the surrounding liver parenchyma. This lesion is isointense to the liver parenchyma in non-enhanced T1- (Fig. g) and T2-weighted MRI (Fig. h). (TIFF 528 kb)

Additional file 3: $\mathrm{MRI}$ of FNH in segment $\mathrm{VI}$ of the liver. Fig. a. Axial T1-weighted non-enhanced MRI shows slightly hypointense focal liver lesion with clearly visible hypointense central scar (arrow). Fig. b. Spin echo sequence, T1-weighted non-enhanced sagittal image presents hypointensive lesion with a central scar. Fig. c. The axial T1-weighted contrast-enhanced image in hepatic arterial phase shows typical intensive homogeneous enhancement of the lesion with a characteristic hypointense central scar. Fig. d. Axial T1-weighted contrast-enhanced MR image: lesion is isointense to the normal liver parenchyma in portal venous phase, the hypointensive central scar is visible (arrow). Fig. e,f. Axial and sagittal images in hepatobiliary phase: lesion is isointense to the surrounding liver parenchyma, the central scar is clearly visible. (TIFF $3002 \mathrm{~kb}$ )

Additional file 4: $C T$ and MRI images of FNH in segment $V$ of the liver. Fig. a,b. CT image in hepatic arterial phase shows typical intensive homogeneous enhancement of the lesion with discreetly visible central scar (arrow). This lesion is hypointense in the non-enhanced T1-weighted image (fig. c) and isointense in the T2-weighted image (fig. d). Fig. e. Axial T1-weighted contrast-enhanced MRI in hepatic arterial phase presents a homogeneous enhancement of the lesion with subtle central scar (arrow). (TIFF 909 kb)

Additional file 5: Results of multiple comparisons of radiological signs in $C T$ by means of McNemar's test. $P$ values presented after BonferroniHochberg's correction. (PDF $108 \mathrm{~kb}$ )

Additional file 6: Results of multiple comparisons of radiological signs in MRI by means of McNemar's test. $P$ values presented after BonferroniHochberg's correction. (PDF $108 \mathrm{~kb}$ )

\section{Abbreviations}

AFROC: Alternative free response receiver operating characteristic; AUC: Area under curve; CS: Central scar; CT: Computed tomography; EP: Equilibrium phase; FLL: Focal liver lesion; FNH: Focal nodular hyperplasia; FOV: Field of view;

FPF: False positive fraction; Gd-BOPTA: Gadobenate dimeglumine; GGTP: Gamma-glutamyltranspeptidase; HAP: Hepatic arterial dominant phase; HBP: Hepatobiliary phase; HCA: Hepatocellular adenoma; HCC: Hepatocellular carcinoma; HH: Hepatic hemangioma; LLF: Lesion location fraction; MDCT: Multidetector computed tomography; MRI: Magnetic resonance imaging; NECT: Non-enhanced computed tomography; NPV: Negative predictive value; PPV: Positive predictive value; Pts: Patients; PVP: Portal venous dominant phase; ROC: Receiver operating characteristic; SD: Standard deviation; T1 W: T1-weighted images; T2 W: T2-weighted images; TE: Echo time; TR: Repetition time

\section{Acknowledgements}

Not applicable.

\section{Funding}

The study has been partially financed from the research grant ST-82 given by authorities of the Medical University of Gdansk to MS.

\section{Availability of data and materials}

The datasets used and analyzed during the current study available from the corresponding author on request. 


\section{Authors' contributions}

TN has substantial contribution to study design, data collection and interpretation and statistical analysis. KM was a major contributor in writing the manuscript. ElS performed the histological examinations and contributed to manuscript preparation. KD has contributed to data collection and interpretation. MS has contributed to study design and data interpretation. ES has contributed to study design, data collection and interpretation and has coordinated research team. All authors read and approved the final manuscript.

\section{Ethics approval and consent to participate}

The research was approved by The Independent Bioethics Committee for Scientific Research of the Medical University of Gdansk, Poland (NKEBN/649/ 01-02). All patients gave their informed written consent to participate.

\section{Consent for publication}

Not applicable.

\section{Competing interests}

The authors declare that they have no competing interests.

\section{Publisher's Note}

Springer Nature remains neutral with regard to jurisdictional claims in published maps and institutional affiliations.

\section{Author details}

'2nd Departement of Radiology, Medical University of Gdansk, Smoluchowskiego 17, 80-214 Gdansk, Poland. '2Department of Pathology and Neuropathology, Medical University of Gdansk, Debinki 1, 80-211 Gdansk, Poland. ${ }^{3}$ Departement of Radiology, Medical University of Gdansk, Debinki 7, 80-211 Gdansk, Poland.

\section{Received: 20 November 2016 Accepted: 29 November 2017}

Published online: 15 January 2018

\section{References}

1. Hirohashi S, Ishak K, Koijro M, Wanless I, Theise N, Tsukuma H, Blum H. Hepatocellular carcinoma. In: Pathology \& Genetics. Tumours of the digestive system. Chapter 8: Tumours of the liver and intrahepatic bile ducts, IARCPress, Lyon, 2000. p. 169

2. Goodman ZD, Terracciano LM. Tumours and tumour-like lesions of the liver. In: Burt AD, Portmann BC, Ferrell LD, editors. MacSween's pathology of the liver. 5th ed. Philadelphia: Churchill Livingstone Elsevier; 2007. p. 761-814.

3. Wanless IR, Mawdsley C, Adams R. On the pathogenesis of focal nodular hyperplasia of the liver. Hepatology. 1985;5:1194-200.

4. Cherqui D, Rahmouni A, Charlotte F, et al. Management of focal nodular hyperplasia and hepatocellular adenoma in young women: a series of 41 patients with clinical, radiological, and pathological correlations. Hepatology. 1995:22:1674-81.

5. Choi CS, Freeny PC. Triphasic helical CT of hepatic focal nodular hyperplasia: incidence of atypical findings. AJR Am J Roentgenol. 1998;170:391-5.

6. Petresein J, Spiazzi A, Giovagnoni A, et al. Focal liver lesions: evaluation of the efficacy of gadobenate dimeglumine in MR imaging - a multicenter phase III clinical study. Radiology. 2000;215:727-36.

7. Grazioli L, Morana G, Federele MP, et al. Focal nodular hyperplasia: morphologic and functional information from MR imaging with Gadobenate Dimeglumine. Radiology. 2001;221:731-9.

8. Grazioli L, Morana G, Kirchin MA, Schneider G. Accurate differentiation of focal nodular hyperplasia from hepatic adenoma at gadobenate dimeglumineenhanced MR imaging: prospective study. Radiology. 2005;236:166-77.

9. Huppertz A, Balzer T. Blakeborough et al. improved detection of focal liver lesions at MR imaging: multicenter comparison of gadoxetic acid-enhanced MR images with intraoperative findings. Radiology. 2004;230:266-75.

10. Pascolo L, Petrovic S, Cupelli F, et al. Abc protein transport of MRI contrast agents in canalicular rat liver plasma vesicle and yeast vacuoles. Biochem Biophys Res Commun. 2001;282:60-6.

11. Tsurusaki M, Sofue K, Murakami T. Current evidence for the diagnostic value of gadoxetic acid-enhanced magnetic resonance imaging for liver metastasis. Hepatol Res. 2016; https://doi.org/10.1111/hepr.12646.

12. Chakraborty DPA. Brief history of free-response receiver operating characteristic paradigm data analysis. Acad Radiol. 2013;20:915-9.
13. International Working Party. Terminology of nodular hepatocellular lesions. Hepatology. 1995:22:983-93.

14. Dev Chakraborty' FROC Webpage. 2005. http://www.devchakraborty.com. Accessed 2 Jan 2018.

15. Fechner RE. Benign hepatic lesions and oral administered contraceptives. Hum Patol. 1977:8:255-68.

16. Brancatelli G, Federele M, Grazioli L, et al. Focal nodular hyperplasia: CT findings with emphasis on multiphasic helical $C T$ in 78 patients. Radiology. 2001;219:61-8

17. Cherqui D, Mathieu D, Zafrani ES, Dhumeaux D. Hyperplasie nodulaire focale et adénome hépatocellulaire chez la femme, Données actuelles. Gastroenterol Clin Biol. 1997;21:929-35.

18. Ferlicot S, Kobeiter H, Tran Van Nhieu J, et al. MRI of atypical focal nodular hyperplasia of the liver: radiology-pathology correlation. AJR Am J Roentgenol. 2004;182:1227-31.

19. Vilgrain V, Flejou JF, Arrive $L$, et al. Focal nodular hyperplasia of the liver: $M R$ imaging and pathologic correlation in 37 patients. Radiology. 1992:84:1-6.

20. Attal P, Vilgrain $V$, Brancatelli $G$, et al. Telangiectatic focal nodular hyperplasia: US, CT, and MR imaging findings with histopathologic correlation in 13 cases. Radiology. 2003;228:465-72.

21. Zech CJ, Grazioli L, Breuer J, Reiser MF, Schoenberg SO. Diagnostic performance and description of morphological features of focal nodular hyperplasia in Gd-EOB-DTPA-enhanced liver magnetic resonance imaging: results of a multicenter trial. Investig Radiol. 2008:43:504-11.

22. Li AJ, Zhou WP, Diagnosis WMC. Treatment of hepatic focal nodular hyperplasia: report of 114 cases. Zhonghua Wai Ke Za Zhi. 2006:44:321-3.

23. Roux M, Pigneur F, Calderaro J, et al. Differentiation of focal nodular hyperplasia from hepatocellular adenoma: role of the quantitative analysis of gadobenate dimeglumine-enhanced hepatobiliary phase MRI. J Magn Reson Imaging. 2015;42:1249-58.

24. Kim YK, Lee JM. Gadobenate dimeglumine-enhanced liver MR imaging: value of dynamic and delayed imaging for the characterization and detection of focal liver lesions. Eur Radiol. 2004:14:5-13.

25. Huppertz A, Haraida S, Kraus A, et al. Enhancement of focal liver lesions at gadoxetic acid-enhanced MR imaging: correlation with histopathologic findings and spiral CT - initial observations. Radiology. 2005;234:468-78.

26. Chung YE, Kim MJ, Kim YE, Park MS, Choi JY, Kim KW. Characterization of incidental liver lesions: comparison of multidetector CT versus Gd-EOBDTPA-enhanced MR imaging. PLoS One. 2013;11(8):e66141. https://doi.org/ 10.1371/journal.pone.0066141.

27. Soussan M, Aubé C, Bahrami S, Boursier J, Valla DC, Vilgrain V. Incidental focal solid liver lesions: diagnostic performance of contrast-enhanced ultrasound and MR imaging. Eur Radiol. 2010;20:1715-25.

28. Sofue K, Tsurusaki M, Murakami T, et al. Does Gadoxetic acid-enhanced 3.0T MRI in addition to 64-detector-row contrast-enhanced CT provide better diagnostic performance and change the therapeutic strategy for the preoperative evaluation of colorectal liver metastases? Eur Radiol. 2014:24:2532-9.

\section{Submit your next manuscript to BioMed Central} and we will help you at every step:

- We accept pre-submission inquiries

- Our selector tool helps you to find the most relevant journal

- We provide round the clock customer support

- Convenient online submission

- Thorough peer review

- Inclusion in PubMed and all major indexing services

- Maximum visibility for your research

Submit your manuscript at www.biomedcentral.com/submit
) Biomed Central 